\title{
Sigmoid volvulus causing intestinal obstruction in a child - A case report and review of literature
}

\begin{abstract}
Introduction: Sigmoid volvulus is a rare cause of intestinal obstruction in pediatric practice. High index of suspicion is necessary to diagnose the condition. Prompt diagnosis and treatment is mandatory to intervene before colon becomes ischaemic and perforates with dire consequences.

Case presentation: We report a case of 11year old Sri Lankan male child who presented to pediatric surgical unit with features suggestive of intestinal obstruction. $\mathrm{X}$ ray abdomen revealed classic omega (coffee bean) sign of sigmoid volvulus. Following resuscitation endoscopic de rotation was attempted, but failed. Subsequently patient underwent laparotomy and Sigmoid volvulus with gangrene and impending perforation was noted. No predisposing cause except long mesentery was identified. Hartmanns procedure with resection of gangrenous bowel was performed at the emergency setting. Patient made an uneventful recovery, and hartmann reversal was performed 8 weeks later. He made a steady recovery and remains free of symptoms to date.
\end{abstract}

Conclusion: Clinicians dealing with pediatric surgical problems should maintain a high degree of suspicion, in order not to miss this important diagnosis, as any delay in instituting treatment has a devastation effect in morbidity as well as mortality. Early diagnosis and prompt treatment confers excellent prognosis.
Volume 2 Issue 2 - 2015

\author{
Weeratunga ND \\ Lady Ridgeway Hospital for children, Sri Lanka
}

Correspondence: Samarakoon LB, Lady Ridgeway Hospital for children, Colombo, Sri Lanka, Tel 947I2726543, Email lasithamfc@gmail.com

Received: March 17, 2015 | Published: April 28, 2015

Keywords: sigmoid, volvulus, paediatric, gangrene, omega, sign hartmanns procedure

\section{Introduction}

Sigmoid volvulus is a common presentation of bowel obstruction in the elderly age group, thought to arise due to redundancy of the Sigmoid colon. ${ }^{1}$ Few cases occurring during pregnancy also have been reported, where enlarging gravid uterus has been implicated in pathaphysiology. ${ }^{2}$ However sigmoid volvulus is a very rare cause of intestinal obstruction in children. There are only very few reported cases to date. ${ }^{3-12}$ We report a case of sigmoid volvulus causing in an 11year old Sri Lankan child, presenting with intestinal obstruction.

\section{Case presentation}

An eleven year old previously healthy Sri Lankan child presented with sudden onset of abdominal pain, to our surgical unit. The pain was colicky in nature at the onset, later becoming constant, had lasted for two days. He complained of abdominal distension initially and progressively developed vomiting and subsequently poor urine output.

Upon examination he was drowsy, dehydrated, and tachypnoeic. $\mathrm{He}$ was also tachycardic. Abdominal examination revealed gross distension with guarding and rigidity. Bowel sounds were absent and digital rectal examination revealed an empty rectum.

Routine blood investigations were performed and were unremarkable. The child was resuscitated with fluids and electrolytes and broad spectrum antibiotics were commenced. Abdominal Xay performed was suggestive of volvulus of the sigmoid colon i.e. Coffee bean sign (Figure 1). Sigmoidoscopic decompression was attempted but was unsuccessful, so it was decided to proceed with emergency laparotomy. Intra operative findings confirmed volvulus of the sigmoid colon with gangrene and impending perforation (Figure 2). Gangrenous segment of the sigmoid colon was resected (Figure 3). We decided against a primary anastomosis and performed a Hartmann's procedure.

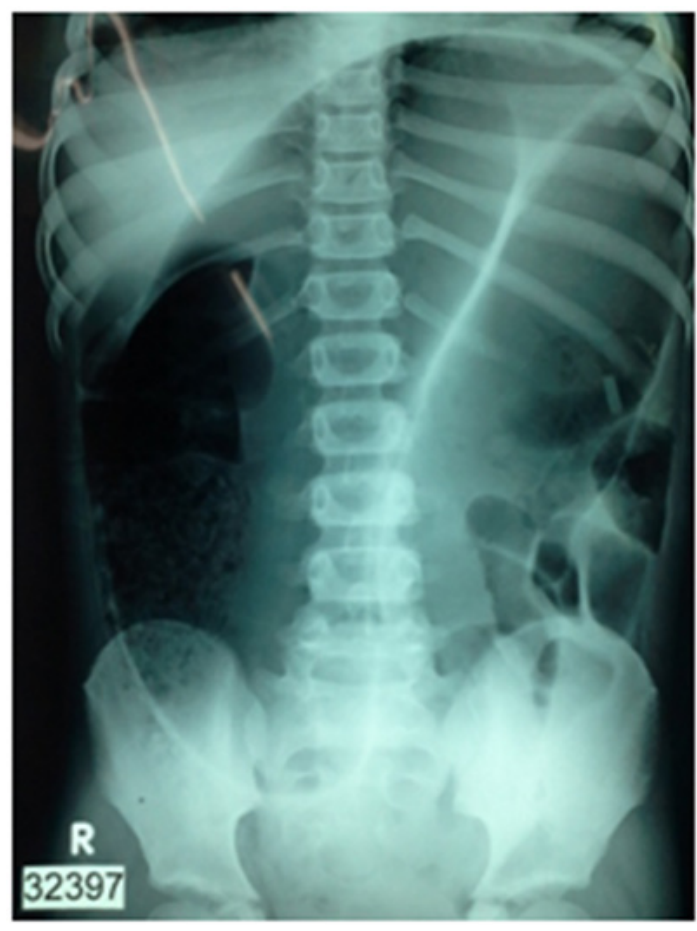

Figure I X-ray Abdomen showing the coffee bean or Omega sign.

Post operatively child was admitted to the intensive care unit for observation. He made an uneventful recovery and was discharged subsequently six days after the surgery. He continued to be followed up at as an outpatient. After eight weeks, Hartmann's procedure was reversed with colo-rectal anastomosis. During subsequent follow-up child made a steady recovery and remains well and asymptomatic 
to this date. Segment of colon that was resected was histologically unremarkable except changes compatible with ischaemic necrosis.

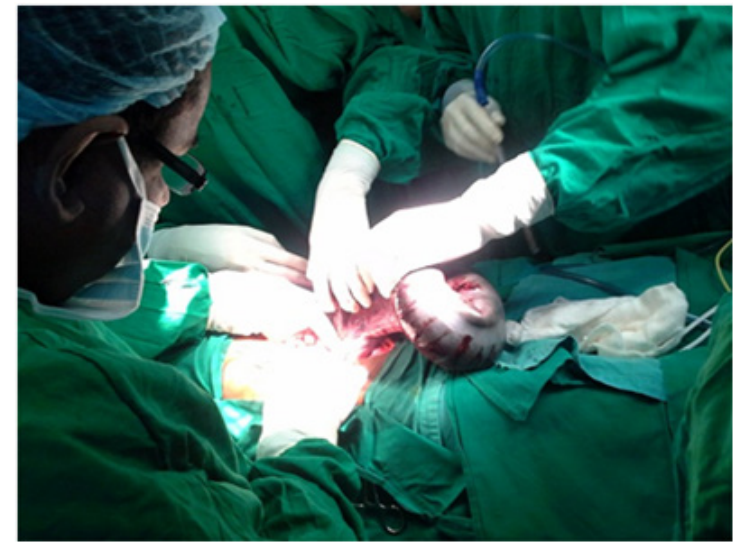

Figure 2 Intraoperative photograph of massively dilated gangrenous loop of sigmoid colon.

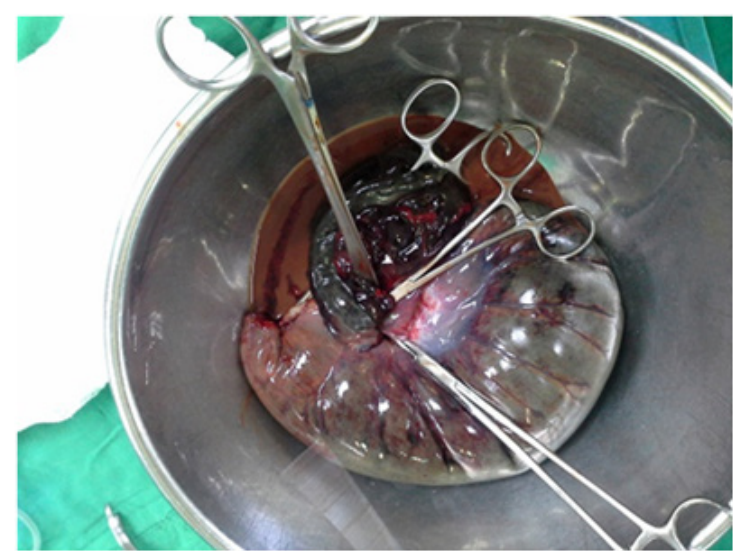

Figure 3 Resected gangrenous loop of sigmoid colon.

\section{Discussion}

Sigmoid volvulus is a common cause of intestinal obstruction in the elderly age group, thought to be due to redundancy of the sigmoid colon loop. Chronic constipation, neuropsychiatric disorders are commonly associated among adults, making the disease common among institutionalized elderly and infirm. ${ }^{1}$ High fiber diet, pregnancy and motility disorders and mental disability have also been implicated. ${ }^{2-4,13-17}$ In particular children with Hirchsprungs disease have been reported with colonic volvulus. ${ }^{18,19}$

In the pediatric surgical practice volvulus of the sigmoid colon remains a rare occurrence. Only few isolated case reports and case series have been reported to date in literature. Salas reported 63 cases of sigmoid volvulus in children in a case series in $2000 .{ }^{20}$ Smith reported 48 cases of sigmoid volvulus in children in a comprehensive review of literature in 1990. ${ }^{3}$ Mellor and Drake in 1994 reported only 14 cases of colonic volvulus in children, with 10 cases of sigmoid volvulus. ${ }^{21}$ Though Sigmoid volvulus is common in India, probably due to high dietary fiber intake, Puneet et al., ${ }^{4}$ reported only six cases of Sigmoid volvulus in children over a period spanning a decade. ${ }^{4}$ $\mathrm{Caeacal}^{22}$ and transverse colonic volvulus, albeit rare, has also been reported in children.

Although volvulus in adults is common in Asian populations, more cases in pediatric practice have been reported from West, including North America. ${ }^{3,21,23}$ Among children several predisposing factors have been identified. Hirchsprungs disease has been implicated in both transverse colonic as well as sigmoid volvulus. In a series reported by Sarioglu, among 10 patients with colonic volvulus due to Hirchsprungs disease, 8 had Sigmoid volvulus. ${ }^{19}$ Short aganglionic segment and dilated ganglionic segment with freely mobile mesentery were thought to be predisposing factors. Congenital anomalous fixation of the colon has been reported as a predisposing factor for sigmoid volvulus. ${ }^{4}$ Two cases of sigmoid volvulus comprising of segmental dilatation of the colon has been reported. ${ }^{24}$ Absence of mesocolon has also been implicated. ${ }^{25}$ Other rarer causes that has been implicated are Prune belly syndrome, ${ }^{3}$ Myotonic dystrophy ${ }^{26}$ and imperforate anus. ${ }^{27}$ Apart from long mesocolon, we could not identify any other predisposing factor in our patient, macroscopically or microscopically in the resected specimen.

In a large series reported by Smith, ${ }^{3}$ mean age of presentation was 8 years. Predisposing factors were present only $33 \%$. Abdominal pain $(66 \%)$, vomiting $(31 \%)$, were the commonest presenting symptoms while abdominal distention $(69 \%)$, tenderness $(41 \%)$ were the commonest signs. Similar findings were also reported from a Nigerian study ${ }^{28}$ Recurrent lower abdominal pain with left lower abdominal tenderness has also been reported. ${ }^{8,10}$ These symptoms were noted in our patient also, although history of recurrent pain was not available. Although classic X ray appearance of coffee bean sign or omega sign was noted in our patient, this was not the norm for other series, with less than a third having this classic radiological sign, with Barium enema reported as having a higher diagnostic yield. ${ }^{3}$ Although common teaching is that" birds beak" appearance is path gnomic, Mellor et al reported that twisted appearance is more common and consistent with a diagnosis of sigmoid Volvulus. ${ }^{21}$ Not only is barium enema diagnostic, it can be therapeutic in uncomplicated cases though it might have to be repeated. In one large series 11 out of 14 colonic volvulus were successfully reduced with barium enema. ${ }^{21}$ Signs of impending perforation with peritonism are a contraindication to enema and mandates urgent surgical intervention as in the patient reported by us.

Definitive treatment of sigmoid volvulus includes resection and anastomosis but this may not be possible as the primary procedure in the emergency setting, where Hartmann's procedure is the preferred alternative. In the absence of peritonism and bowel ischaemia, a trial of sigmoidoscopic deroatation and insertion of rectal tube with elective resection later once to patient is stabilized is feasible. ${ }^{29-31}$ Primary deroatation itself is not an acceptable alternative due to high recurrence rate. ${ }^{3,32}$ Following primary endoscopic deroatation laparoscopic Sigmoid colectomy has also been reported. ${ }^{33}$ Although various procedures to prevent recurrence including extraperitonalization, sigmoidopexy and mesosigmoidoplasty has been proposed in adults, ${ }^{4,34-37}$ their role in routine pediatric surgical practice outside specialist centers remains unclear.

Provided it is diagnosed early and treated promptly, prognosis of volvulus of sigmoid colon is excellent. Mortality rates of 14 percent has been reported, mostly in high risk patients, very young, and patients with other associated anomalies ${ }^{11}$ in one series and 21 percent in another series, ${ }^{28}$ mostly due to sepsis.

\section{Conclusion}

Sigmoid volvulus is a rare presentation of intestinal obstruction in children. Clinicians dealing with pediatric surgical problems should maintain a high degree of suspicion, in order not to miss this important diagnosis, as any delay in instituting treatment has a devastation effect in morbidity as well as mortality. Early diagnosis and prompt treatment confers excellent prognosis. 


\section{Acknowledgements}

The authors would like to acknowledge Intern Medical Officers Dr. Nuwan Padumadasa, Dr. Shashika Jayasundara, Dr. Shakira Banu for their contributions in the overall management of the patient.

\section{Consent}

Written informed consent was obtained from the patient and parents for publication of this case report and any accompanying images. A copy of the written consent is available for review by the Editor-in-Chief of this journal.

\section{Conflicts of interest}

Author declares there are no conflicts of interest.

\section{Funding}

None.

\section{References}

1. Ballantyne GH, Brandner MD, Beart RW, et al. Volvulus of the colon Incidence and mortality. Ann Surg. 1985;202(1):83-92.

2. Samarakoon L, Weerasekera A, Sanjeewa R, et al. Sigmoid volvulus causing intestinal obstruction in pregnancy. Journal of symptoms and signs. 2013;2(2):4.

3. Smith SD, Golladay ES, Wagner C, et al. Sigmoid volvulus in childhood South Med J. 1990;83(7):778-781.

4. Puneet, Khanna R, Gangopadhyay AN, et al. Sigmoid volvulus in childhood: report of six cases. Pediatr Surg Int. 2000;16(1-2):132-133.

5. Atamanalp SS, Yildirgan MI, Basoglu M, et al. Sigmoid colon volvulus in children: review of 19 cases. Pediatr Surg Int. 2004;20(7):492-495.

6. Campbell JR, Blank E. Sigmoid volvulus in children. Pediatrics 1974;53(5):702-705.

7. Carter R, Hinshaw DB. Acute sigmoid volvulus in children. Am J Dis Child. 1961;101(5):631-634.

8. Cook ES, Allison EJ. Sigmoid volvulus in a young patient. Ann Emerg Med. 1984;13(10):963-966.

9. Hunter JG, Keats TE. Sigmoid volvulus in children. A case report. The American journal of roentgenology, radium therapy, and nuclear medicine. 1970;108(3):621-623.

10. Krupsky S, Halevy A, Orda R. Sigmoid volvulus in adolescence. J Clin Gastroenterol. 1987;9(4):467-469.

11. McCalla TH, Arensman RM, Falterman KW. Sigmoid volvulus in children. The American surgeon. 1985;51(9):514-519.

12. Wilk PJ, Ross M, Leonidas J. Sigmoid volvulus in an 11-year-old girl. Case report and literature review. Am J Dis Child. 1974;127(3):400-402.

13. Iwamoto I, Miwa K, Fujino T, et al. Perforated colon volvulus coiling around the uterus in a pregnant woman with a history of severe constipation. J Obstet Gynaecol Res . 2007;33(5):731-733.

14. Joshi MA, Balsarkar D, Avasare N, et al. Gangrenous sigmoid volvulus in a pregnant woman. Trop Gastroenterol . 1999;20(3):141-142.

15. Lazaro EJ, Das PB, Abraham PV. Volvulus of the sigmoid colon complicating pregnancy. Obstet Gynecol . 1969;33(4):553-557.
16. Atamanalp SS, Öztur G. Sigmoid volvulus in pregnancy. Turk J Med Sc. 2012;42(1):9-15.

17. Takada K, Hamada Y, Sato M, et al. Cecal volvulus in children with mental disability. Pediatr Surg Int. 2007;23(10):1011-1014.

18. Neilson IR, Youssef S. Delayed presentation of Hirschsprung's disease: acute obstruction secondary to megacolon with transverse colonic volvulus. J Pediatr Surg. 1990; 25(11):1177-1179.

19. Sarioglu A, Tanyel FC, Buyukpamukcu N, et al. Colonic volvulus: a rare presentation of Hirschsprung's disease. J Pediatr Surg. 1997;32(1):117-118.

20. Salas S, Angel CA, Salas N, et al. Sigmoid volvulus in children and adolescents. J Am Coll Surg. 2000;190(6):717-723.

21. Mellor MF, Drake DG. Colonic volvulus in children: value of barium enema for diagnosis and treatment in 14 children. AJR Am J Roentgenol. 1994;162(5):1157-1159.

22. Kirks DR, Swischuk LE, Merten DF, et al. Cecal volvulus in children. AJR American journal of roentgenology. 1981;136(2):419-422.

23. Howell HS, Freeark RJ, Bartizal JF. Transverse colon volvulus in pediatric patients. Arch Surg. 1976;111(1):90.

24. Ravasse P, Petit T, Cau D, et al. Volvulus of the sigmoid colon as a complication of segmental dilatation of the colon. Report of 2 cases. Eur J Pediatr Surg. 1996;6(6):375-377.

25. Srouji MN, Finnigan LP, Boas RN. Neonatal sigmoid volvulus with absence of mesocolon. J Pediatr Surg. 1974;9(5):779-781.

26. Houshian S, Sorensen JS, Jensen KE. Volvulus of the transverse colon in children. J Pediatr Surg . 1998;33(9):1399-1401.

27. Janik JS, Humphrey R, Nagaraj HS. Sigmoid volvulus in a neonate with imperforate anus. J Pediatr Surg. 1983;18(5):636-638.

28. Ameh EA, Nmadu PT. Intestinal volvulus: aetiology, morbidity, and mortality in Nigerian children. Pediatr Surg Int . 2000;16(1-2):50-52.

29. Ghazi A, Shinya H, Wolfe WI. Treatment of volvulus of the colon by colonoscopy. Ann Surg. 1976;183(3):263-265.

30. Yang YJ, Chang MH, Ni YH. Sigmoid volvulus in children: report of two cases. J Formos Med Assoc. 2001;100(2):134-136.

31. Seger DL, Middleton D. Childhood sigmoid volvulus. Annals of emergency medicine. 1984;13(2):133-135.

32. Ismail A. Recurrent colonic volvulus in children. J Pediatr Surg. 1997;32(12):1739-1742.

33. Liu KK, Leung MW, Wong BP, et al. Minimal access surgery for sigmoid volvulus in children. Pediatr Surg Int. 2006;22(12):1007-1008

34. Bhatnagar BN. Extraperitonealization of sigmoid for prevention of recurrence of volvulus: a follow-up study. Am $J$ Protocol. 1977;28(1):37-44

35. Bhatnagar BN, Roy SK, Chakravarty MR. Comparative evaluation of the commonly performed emergency procedures for the prevention of recurrence of sigmoid volvulus. Am J Protocol. 1977;28(5):35-44.

36. Ponticelli A, Mastrobuono I, Matarazzo E, et al. Mesosigmoidoplasty in the treatment of sigmoid volvulus in children. S Afr J Surg. 1989;27(3):105-107.

37. Bhatnagar BN. Prevention of recurrence of sigmoid colon volvulus: a new approach. A preliminary report. $J$ R Coll Surg Edinb. 1970;15(1):49-52. 